\title{
RETRACTED ARTICLE: A low delay multi-source multicast algorithm for MANETs
}

\author{
Yinbo Zuo $\cdot$ Xianggao Cai $\cdot$ Xiaola Lin
}

Published online: 13 September 2013

(C) Springer Science+Business Media New York 2014

The publisher has retracted this article, after determining that the author failed to cite properly: "A Depth-optimal Lowcomplexity Distributed Wireless Multicast Algorithm", by
A.S. Akyurek, published in 2010 in "MILCOM", DOI 10.1109/MILCOM.2010.5680407, and in 2011 in "Oxford Journals - Computer Journal”. 